\title{
Analysis of earthquake body wave spectra for potency and magnitude values: implications for magnitude scaling relations
}

\author{
Zachary E. Ross, ${ }^{1, *}$ Yehuda Ben-Zion, ${ }^{1}$ Malcolm C. White ${ }^{2}$ and Frank L. Vernon ${ }^{2}$ \\ ${ }^{1}$ Department of Earth Sciences, University of Southern California, Los Angeles, CA 90089-0740, USA. E-mail: zross@gps.caltech.edu \\ ${ }^{2}$ Scripps Institution of Oceanography, University of California, San Diego, CA 92093-0225, USA
}

Accepted 2016 August 31. Received 2016 August 22; in original form 2016 March 23

\begin{abstract}
SUMMAR Y
We develop a simple methodology for reliable automated estimation of the low-frequency asymptote in seismic body wave spectra of small to moderate local earthquakes. The procedure corrects individual $P$ - and $S$-wave spectra for propagation and site effects and estimates the seismic potency from a stacked spectrum. The method is applied to $>11000$ earthquakes with local magnitudes $0<M_{\mathrm{L}}<4$ that occurred in the Southern California plate-boundary region around the San Jacinto fault zone during 2013. Moment magnitude $M_{\mathrm{w}}$ values, derived from the spectra and the scaling relation of Hanks \& Kanamori, follow a Gutenberg-Richter distribution with a larger $b$-value (1.22) from that associated with the $M_{\mathrm{L}}$ values (0.93) for the same earthquakes. The completeness magnitude for the $M_{\mathrm{w}}$ values is 1.6 while for $M_{\mathrm{L}}$ it is 1.0. The quantity $\left(M_{\mathrm{w}}-M_{\mathrm{L}}\right)$ linearly increases in the analysed magnitude range as $M_{\mathrm{L}}$ decreases. An average earthquake with $M_{\mathrm{L}}=0$ in the study area has an $M_{\mathrm{W}}$ of about 0.9 . The developed methodology and results have important implications for earthquake source studies and statistical seismology.
\end{abstract}

Key words: Earthquake source observations; Seismicity and tectonics; Body waves; Statistical seismology.

\section{INTRODUCTION}

The size of an earthquake is quantified with different parameters. One basic measure of the earthquake size is the scalar seismic potency $P_{0}$ given by the integral of the slip over the failure area or more generally the integral of inelastic strain over the source volume (e.g. Ben-Zion 2003; Ben-Menahem \& Singh 2012). A related more commonly used parameter is the scalar seismic moment, $M_{0}$, given by the product of the potency and effective rigidity in the source volume. The seismic potency and moment are proportional to the low-frequency asymptote of the displacement source spectrum (e.g. Aki 1966; Brune 1970; Ben-Zion 2003). In addition to quantifying the size of earthquakes, the seismic potency/moment is also important for deriving many other source properties including stress/strain drop and radiated seismic energy (e.g. Aki \& Richards 2002; Prieto et al. 2004).

Estimating $P_{0}$ for small earthquakes is non-trivial because the wavefield recorded by a seismometer is affected by additional factors beyond the source radiation. It requires knowledge of how

\footnotetext{
* Now at: Seismological Laboratory, California Institute of Technology, Pasadena, CA 91125, USA.
}

propagation and site, and recording conditions have altered a given spectrum so that they can be corrected for (e.g. Abercrombie 1995; Edwards et al. 2010). Accounting for propagation effects requires a robust estimate of an earthquake's location. The final step of extracting the long-period asymptote generally requires fitting a parametrized model to the corrected spectra, which has its own uncertainties. Small earthquakes are further susceptible to bandwidth limitations of the recording system.

In contrast, the local magnitude, $M_{\mathrm{L}}$, requires only an estimate of the source receiver distance and a measurement of the peak amplitude of a seismogram filtered with the instrument response of a Wood-Anderson seismograph (Richter 1935). It is a far more stable measurement for small to moderate events as it only depends on two variables. This likely contributed to its longevity; it is still regularly used by seismic networks, even at the present day. However, $M_{\mathrm{L}}$ is not tied directly to a physical source property (e.g. Deichmann 2006), so is only useful for quantifying relative differences between events; for large earthquakes $M_{\mathrm{L}}$ becomes saturated and is no longer useful. Various studies have tried to develop scaling relationships between $M_{\mathrm{L}}$ and $M_{0}$ or $P_{0}$. The best-known relation was developed by Hanks \& Kanamori (1979) for moderate to large earthquakes and led to the moment magnitude scale $M_{\mathrm{w}}$. Several later studies (e.g. Bakun 1984; Hanks \& Boore 1984; Abercrombie 1996; 
Ben-Zion \& Zhu 2002; Shearer et al. 2006; Edwards et al. 2010) noted the apparent deviation of the $M_{\mathrm{L}}$ and $M_{\mathrm{W}}$ scales for magnitudes below $\sim 3.5$. These studies analysed events having $M_{\mathrm{L}}$ above about zero.

Earthquakes are well known to be exponentially distributed with respect to magnitude (Gutenberg \& Richter 1944). The slope of the logarithm of this distribution is commonly referred to as the $b$-value. The $b$-value quantifies the relative frequency of events of different sizes and has been used widely in many applications (e.g. Utsu 1999; Ben-Zion 2008). Abercrombie (1996) found that the $b$-value of data recorded in a deep borehole is constant down to at least $M_{\mathrm{L}}=0$. Given that the $M_{\mathrm{w}}$ and $M_{\mathrm{L}}$ scales diverge for small events, it is important to compare the $b$-values and completeness magnitudes of earthquakes quantified by both the different scales.

In this study, we calculate seismic potency/moment and $M_{\mathrm{w}}$ values for $>11000$ small to moderate earthquakes which occurred in the Southern California plate-boundary region around the San Jacinto fault zone (SJFZ) in 2013. The phase picks, event detections and spectral fitting process are entirely automated, providing an objective and systematic set of results. In agreement with previous studies, the $M_{\mathrm{L}}$ and $M_{\mathrm{w}}$ scales are shown to diverge for $M<3.5$. The results indicate systematic differences in the $b$-values and completeness magnitudes of two different scales for the same data, making these dependent on the type of magnitude used. A scaling relationship is developed between $M_{\mathrm{L}}$ and $M_{\mathrm{w}}$ that is suitable for converting between magnitudes of events in Southern California.

\section{DATA}

We analyse more than 13570 earthquakes that occurred in 2013 and listed in a recently produced seismicity catalogue for the region around the SJFZ (Ross et al. 2016). The catalogue was built from scratch using the raw waveform archives. In addition to locations, the catalogue contains $223938 P$-wave arrival picks and 199647 $S$-wave arrival picks. The $P$-wave picks are made using the ratio of a short-term moving average to a long-term moving average (e.g. Allen 1978, 1982), while the $S$-wave picks are made using the algorithm of Ross \& Ben-Zion (2014), and updated by Ross et al. (2016). These picks and the hypoDD method (Waldhauser \& Ellsworth 2000) are used to obtain high-quality locations and origin times. The resulting relocated events (totaling 11 175) are compared with the SCSN catalogue (SCEDC 2013; scedc.caltech.edu) to find common events (8082). The local magnitudes of these events are taken from the SCSN catalogue. The potency values are derived from waveform data comprised of $\mathrm{HH}, \mathrm{EH}$ and $\mathrm{HN}$ channels at 87 stations. In the following analysis, each event is required to have at least $5 P$ and $5 S$-wave picks to ensure sufficient quality of the derived potency values. We do not impose any requirements on azimuthal gaps because the events in the SJFZ are generally well covered (Fig. 1) and the emphasis of this study is on statistical results associated with a large data set. The 2013 earthquakes satisfying these criteria are shown in Fig. 1.

\section{METHODS}

A commonly used model for the spectrum of a seismic source is parametrized by a corner frequency, $f_{\mathrm{c}}$, low-frequency asymptote, $\Omega_{0}$ and spectral fall-off, $n$ (e.g. Abercrombie 1995),

$$
A(f)=\frac{\Omega_{0} \cdot \exp \left(-\pi f t^{*}\right)}{1+\left(f / f_{c}\right)^{n}}
$$

where $A(f)$ is the displacement amplitude spectrum, $t^{*}$ is the wholepath attenuation operator and $f$ is frequency. The seismic potency is proportional to $\Omega_{0}$. Estimating $\Omega_{0}$ for small earthquakes is difficult because of many issues. For example, there is often complex attenuation along the path and especially near the surface (e.g. Kilb et al. 2012; Liu et al. 2015), and the spectra are susceptible to noise issues which can affect the usable frequency range (Edwards et al. 2010; Kwiatek \& Ben-Zion 2016) and random heterogeneities (Shearer et al. 2006).

For each available event and station, the mean value for each component was subtracted from the raw data and corrected for instrument response. We then obtain signal and noise windows that are $1.25 \mathrm{~s}$ in duration. The signal window starts $0.25 \mathrm{~s}$ before the pick, while the noise window ends $2 \mathrm{~s}$ before the $P$-wave pick. We tested window lengths in the range 1.0-2.0 s, and found that the results did not change significantly. A multi-taper algorithm is used (Thomson 1982; Park et al. 1987) to calculate amplitude spectra for each of the three components for both $P$ and $S$ waves. The spectra are re-sampled in the $\log$ domain with $\Delta \log (f)=0.05$ (e.g. Ide et al. 2003) and the three-component spectra are combined via,

$D=\sqrt{N^{2}+E^{2}+Z^{2}}$

where $D$ represents the vector amplitude spectrum and $N, E$ and $Z$ are the north, east and vertical components, respectively. From here on, references to spectra are in the form of (2). Signal-to-noise ratio (SNR) spectra are obtained for each station-event pair by squaring the signal and noise spectra. For subsequent analysis, we use only spectra that have at least 75 per cent of the SNR spectral amplitudes above 5.0. If fewer than 5 spectra are left for either $P$ or $S$ waves, the event is skipped. For the remaining events, spectra are corrected for radiation pattern using an average value 0.52 for $P$ waves and 0.63 for $S$ waves (Boore \& Boatwright 1984). At this point, all remaining spectra are integrated to displacement and then corrected for propagation and site effects. These correction factors include geometric spreading, path-dependent attenuation $\left(t^{*}\right)$, as well as a free-surface correction of 2.0. This is a commonly used average value (e.g. Edwards et al. 2010), although in detail the free-surface correction depends on the incidence angle. For source-receiver distances less than $20-30 \mathrm{~km}$, the error in the average value over all stations should generally translate into $\sim 0.1$ magnitude units or less. We calculate $t^{*}$ for each source-receiver combination by tracing rays using a 1-D version of the Allam \& Ben-Zion (2012) velocity model (Table S1, Supporting Information). For these calculations, we use $Q$ values for $P$ and $S$ waves from Abercrombie (1997) for depths less than $2.5 \mathrm{~km}$ and $Q=1000$ at depths larger than $2.5 \mathrm{~km}$ (Table $\mathrm{S} 1$, Supporting Information).

The described steps result in a set of $P$ - and $S$-wave displacement source spectra for each earthquake. Next, we stack each event's spectra for each phase type separately (Fig. 2). The stacking is done by calculating the median logarithmic spectral value at each frequency bin rather than the mean. This keeps the stack from being biased by outlier spectra, without the need to discard them. We also stack the individual SNR spectra together to get an estimate of the average SNR at each frequency. This stacking is done in the $\log (\mathrm{SNR})$ domain to keep the closest stations from dominating the result (Fig. 2). The stacked SNR spectrum is used to determine the usable frequency range for each phase stack automatically. While this range varies between different stations for a given event, the stacked SNR spectrum was found to be an excellent indicator of where artefacts began to appear in the stack. The artefacts at the low frequencies tend to cause the displacement spectra to tail 



Figure 1. Map of the San Jacinto fault zone region and >13 000 earthquakes which occurred during 2013, detected and located by Ross et al. (2016). Blue triangles indicate stations used. The yellow star is the location of the example event in Fig. 2. The bottom panel shows a seismicity profile along the strike of the San Jacinto fault zone.

upward rather than become asymptotic. A lower frequency bound, $f_{\mathrm{L}}$, is chosen to be the frequency value at the left end of the spectrum at which SNR > 5.0 was first satisfied. An upper frequency bound, $f_{\mathrm{H}}$, is chosen in the same manner while searching from the right, but is limited to a maximum value of $40 \mathrm{~Hz}$ due to peaks in the response of broad-band seismometers above this value. Examples of the SNR stack and frequency range are shown in Fig. 2. This imposes a minimum event size with a corner frequency of $P$ wave less than $40 \mathrm{~Hz}$, which translates roughly into a moment magnitude of $\sim 0.7$.

Next, the source model (1) is fit separately to each stacked phase spectrum for a given event. This is performed using a grid search minimizing the sum of the squared logarithm of the residuals. Using the logarithm of the residuals keeps the low frequencies from dominating the sum of squares. The search range used for $\Omega_{0}$ is $[0.75 p$,
$1.25 p$ ] where $p$ is the peak of the stacked displacement spectrum. The search range for the corner frequency $\left[f_{\mathrm{L}}, f_{\mathrm{H}}\right]$ and the search range for $n$ is $[1.5,3.0]$. The seismic potency is proportional to $\Omega_{0}$ (e.g. Aki \& Richards 2002; Ben-Zion 2003),

$P_{0}=4 \pi \Omega_{0} v_{s}$,

$P_{0}=4 \pi \Omega_{0} v_{p}^{3} / v_{s}^{2}$,

where $v_{S}$ and $v_{P}$ are $P$ and $S$ seismic velocities based on a 1-D version of the tomography model of Allam \& Ben-Zion (2012), and eqs (3a) and (3b) are used for the $S$ and $P$ spectra, respectively. For each event, the velocities are taken at the hypocentral depth. Examples of best-fitting spectral results are given in Fig. 2 for the event indicated by a yellow star in Fig. 1 . The final potency value calculated for a given event is derived from a weighted average of 

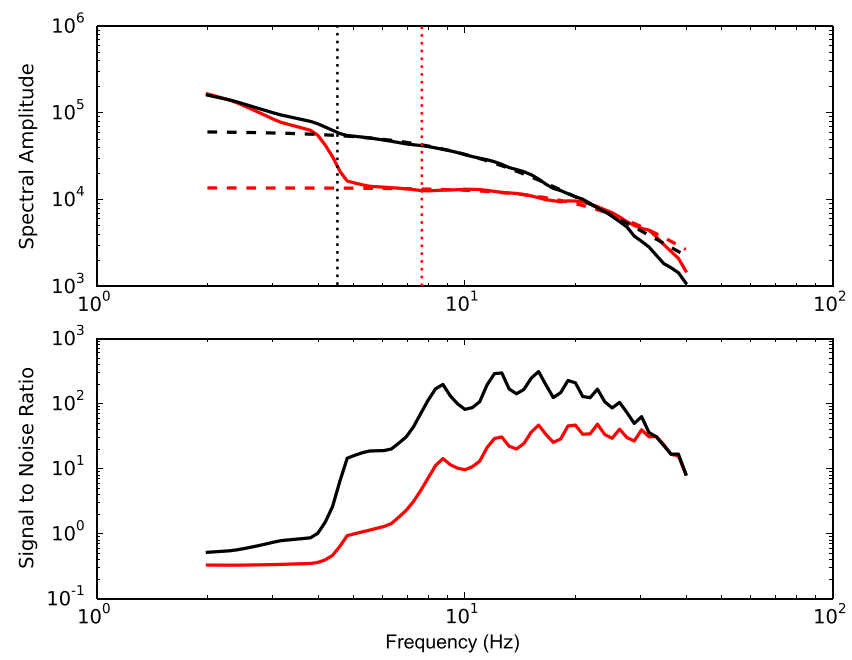

Figure 2. Demonstration of the spectral fitting process for event 15269481 (SCSNID; yellow star, Fig. 1) based on $10 P$ and $10 S$ waves. The upper panel shows stacked spectra for $P$ (red) and $S$ (black) waves. The horizontal dashed lines are the best-fitting source models. The lower panel shows the stacked SNR spectra for $P$ and $S$ waves. The frequency ranges used for the fitting process are the spectral values to the right of the vertical dotted lines (upper panel), which were determined from a threshold of SNR $=5$. The upward tailing observed in the spectral amplitudes to the left of the dotted lines is an artefact of low SNR. The determined moment magnitude for this earthquake is 1.35 , while the $\operatorname{SCSN} M_{\mathrm{L}}$ is 0.90 .

the values obtained for the $P$ and $S$ waves, with the weights inversely related to the number of phases used. The obtained seismic potency value is then converted to moment by assuming a nominal rigidity of $30 \mathrm{GPa}$, and used to calculate $M_{\mathrm{w}}$ with the scaling relation of Hanks \& Kanamori (1979). In this study, we focus on developing and implementing an efficient and robust procedure for estimating source spectral levels and parameters that are included in typical earthquake catalogues. Analysing corner frequencies and spectral decay exponents is outside the goals of this work and requires additional analysis procedures (e.g. Abercrombie 1995; Ross \& Ben-Zion 2016).

\section{RESULTS}

The discussed spectral fitting algorithm was applied to 11175 earthquakes from the Ross et al. (2016) catalogue. The method was able to calculate potency values for 11091 events. Of these events, 8078 were also detected by the SCSN, which form the final subset we use for analysis. Fig. 3 shows frequency-size statistics of the obtained results using for event size both $M_{\mathrm{w}}$ derived from our results (top panel) and $M_{\mathrm{L}}$ values taken from the SCSN catalogue (bottom panel). For both magnitude scales, the results generally follow the Gutenberg-Richter statistics,

$\log (N)=a-b M$,

where $a$ and $b$ are coefficients to be estimated and $M$ is the magnitude. To estimate the $b$-values of the results in Fig. 3, we manually estimate the magnitude of completeness based on a change of slope and find values of 1.0 and 1.6 (vertical blue lines) for the $M_{\mathrm{L}}$ and $M_{\mathrm{w}}$ scales, respectively. Using these values, we estimate the $b$-value for the data with the maximum likelihood method (Aki 1965) and obtain values of 0.93 and 1.16 for the $M_{\mathrm{L}}$ and $M_{\mathrm{w}}$ scales, respectively. The magnitude values in the $M_{\mathrm{w}}$ scale are generally shifted to the right (increased), but the shift is not constant for all magnitudes
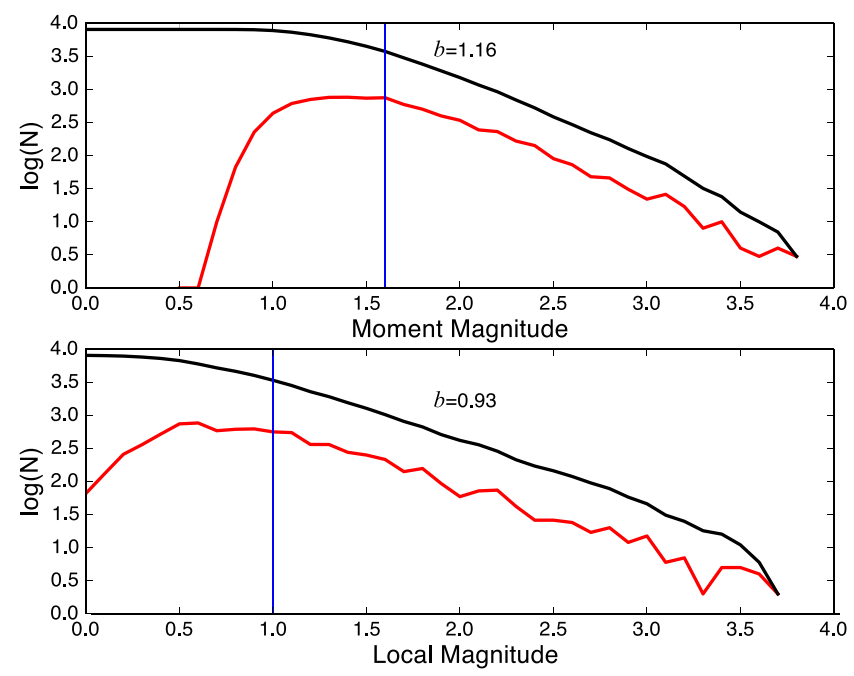

Figure 3. Frequency-magnitude distributions (FMD) for $M_{\mathrm{W}}$ and $M_{\mathrm{L}}$ values of the same data. In total, 8078 of the 11091 events with moment magnitudes were also detected by the SCSN. Only these common events are used. Cumulative and non-cumulative FMD are shown as black and red lines, respectively. The bin spacing for both plots is 0.1 units. The $M_{\mathrm{W}}$ results have a $b$-value of 1.16 , while the $M_{\mathrm{L}}$ results have a $b$-value of 0.93 . From bootstrap resampling, the uncertainty in $b$-value for $M_{\mathrm{w}}$ is estimated to be 0.058 . The completeness magnitude for $M_{\mathrm{w}}$ is estimated at 1.6 , while for $M_{\mathrm{L}}$ it is 1.0 (blue lines). Note that since the same events are in the top and bottom panels, the larger magnitudes produce a $b$-value increase rather than a decrease.

leading to a $b$-value that is about 25 per cent higher. In contrast to many studies that compare $b$-values between different samples of events, the change of $b$-value in Fig. 3 results solely from using the two different magnitude scales for the same events. Bootstrap resampling of the $M_{\mathrm{W}}$ values indicates that the uncertainty in the $b$-value estimates is below 0.06 .

More direct results on the relationship between the $M_{\mathrm{L}}$ and $M_{\mathrm{w}}$ scales for our data set are shown in Fig. 4. There are few events with $M_{\mathrm{L}}>4.0$ and few values with $M_{\mathrm{L}}<0$, so we focus on this magnitude range. A scatterplot of $\log \left(P_{0}\right)$ against $M_{\mathrm{L}}$ is presented in Fig. $4 \mathrm{a}$ along with a best-fit line (red) having a slope of 1.13 (Table 1). This is similar to the value obtained by Ben-Zion \& Zhu (2002) and other studies analysing small events, and significantly different from the 1.5 value in the scaling relation of Hanks \& Kanamori (1979) characterizing moderate and large events. Examination of $\left(M_{\mathrm{w}}-M_{\mathrm{L}}\right)$ vs $M_{\mathrm{L}}$ shows that the magnitude scales are approximately equal only at M3.5 (Fig. 4b), and that for $M_{\mathrm{L}}<3.5,\left(M_{\mathrm{w}}-M_{\mathrm{L}}\right)$ increases linearly with decreasing magnitude. Bootstrap resampling indicates that the uncertainty in the slope is 0.078 . At $M_{\mathrm{L}} 0$, the average earthquake has an $M_{\mathrm{w}}$ value that is 0.88 units larger. From this data set, we also calculate a best-fitting line (Table 1). The standard deviation of the residuals is 0.19 units. The best-fitting values in Table 1 can be used to convert local magnitudes into moment magnitudes (or potency) for earthquakes in Southern California. However, due to regional differences in attenuation, these values are not likely applicable elsewhere.

\section{DISCUSSION}

We derived potency estimates for $>11000$ earthquakes with $0<M_{\mathrm{L}}<4$ around the SJFZ from spectral analysis of both $P$ and $S$ waves. The potency calculations were entirely automated, involving as many as 85 stations in estimating each value of $P_{0}$, 

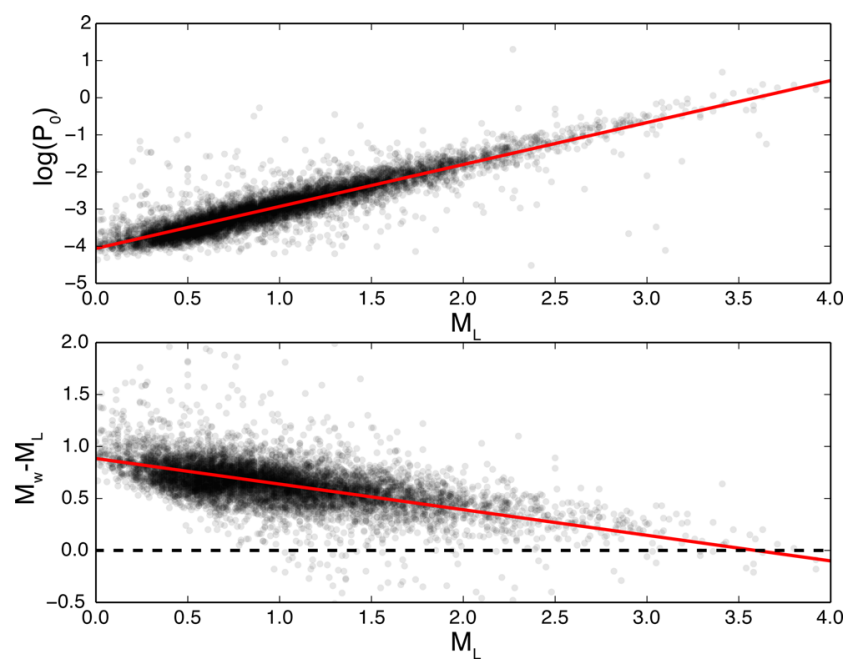

Figure 4. Comparison of derived potency, $M_{\mathrm{W}}$ and $M_{\mathrm{L}}$ values for more than 8000 earthquakes which occurred during 2013. The $M_{\mathrm{L}}$ values were determined by SCSN, while $M_{\mathrm{w}}$ values were determined with the developed procedure. The slope of the best-fitting line (red) in the upper panel is 1.13, significantly different from the 1.5 slope of Hanks \& Kanamori (1979). The uncertainty in the slope is estimated to be 0.078 . The $M_{\mathrm{W}}$ and $M_{\mathrm{L}}$ values are roughly the same around $M_{\mathrm{L}} 3.5$, but below this magnitude $M_{\mathrm{w}}$ progressively increases relative to $M_{\mathrm{L}}$. For $M_{\mathrm{L}}=0$, the average $M_{\mathrm{w}}$ is 0.88 units larger. A linear fit between $M_{\mathrm{w}}$ and $M_{\mathrm{L}}$ over the examined magnitude range leads to the relation $M_{\mathrm{W}}=0.754 M_{\mathrm{L}}+0.88$.

Table 1. Magnitude scaling relation parameters.

\begin{tabular}{lccc}
\hline Quantity & $\mathrm{c}$ & $\mathrm{d}$ & $\sigma$ \\
\hline$P_{0}$ & 1.13 & -4.06 & 0.29 \\
$M_{\mathrm{w}}-M_{\mathrm{L}}$ & -0.246 & 0.884 & 0.19 \\
\hline
\end{tabular}

The calculated values were obtained by linear least-squares regression $\left(y=c M_{\mathrm{L}}+d\right)$.

which leads to a more objective and robust set of results. While the estimates of potency may be improved by manual adjustment of frequency ranges, window lengths, and other various parameters, this is labour intensive and difficult to perform for more than a few hundred events. The effect of attenuation on the lowfrequency asymptotes is generally not appreciable. We performed the same analysis using $Q=1000$ at all depths and found that the results were largely unchanged, with only slightly lower potency values (leading to less than $0.1 M_{\mathrm{w}}$ difference on average). However, using more realistic estimates of $Q$ in the upper $2.5 \mathrm{~km}$ from Abercrombie (1997) made the results between $P$ and $S$ waves agree better, supporting the need for including near-surface attenuation in the calculations.

The zero-frequency asymptote used to derive potency values for the analysed earthquakes is one of three parameters that are estimated from the spectra in our procedure. While some trade-off may occur between the zero-frequency asymptote and $f_{\mathrm{c}}$, it is much less of an issue than between $f_{\mathrm{c}}$ and $n$. This is because the zero-frequency asymptote is estimated from the lowest usable frequencies in the spectrum, while $f_{\mathrm{c}}$ is measured from generally much higher frequencies. From our testing, even if $f_{\mathrm{c}}$ is somewhat-incorrectly estimated, the potency determination typically remains stable. The parameters $f_{\mathrm{c}}$ and $n$ are more strongly related, however, since the value of $f_{\mathrm{c}}$ determines where the high frequencies begin, and thus the rate at which they fall-off.

The results in Fig. 4 confirm previous findings that the scaling relation between the potency (or moment) and local magnitude of events with $M_{\mathrm{L}}<3.5$ strongly deviates from the relation of Hanks \& Kanamori (1979) for moderate and large events. The magnitude of completeness and $b$-value of events with size measured by $M_{\mathrm{L}}$ differ from the values of the same events with size measured by $M_{\mathrm{w}}$. This is important to note because numerous studies in the literature have discussed variations of $b$-value with space and time with no attention to the scale used to measure the magnitudes (e.g. Gerstenberger et al. 2001). Similarly, some studies have compared $b$-values across broad magnitude ranges ignoring the different scales used. The derived scaling relations between $\log \left(P_{0}\right), M_{\mathrm{L}}$ and $M_{\mathrm{w}}$ (Table 1) can be used to convert event size from one scale to the others. We note that this is strictly appropriate for the region around southern California where the analysed events occurred, since regional changes in attenuation can affect the $M_{\mathrm{L}}$ values. However, the overall similar slopes for small events between $\log \left(P_{0}\right)$ or $\log \left(M_{0}\right)$ and $M_{\mathrm{L}}$ found in other studies (e.g. Bakun 1984; Abercrombie 1996; Ben-Zion \& Zhu 2002; Shearer et al. 2006; Edwards et al. 2010) indicate that the regional variations are considerably smaller than changes in the scaling of events with $M_{\mathrm{L}}$ less than about 3 and moderate to large events (e.g. $M_{\mathrm{L}}>4$ ).

The significant scatter in Fig. 4 reflects the difficulties in calculating potency and moment values for small events. Measuring $P_{0}$ requires a high-quality location, phase picks for both phases at many stations, precise estimates of the path and site contributions to a spectrum and a robust technique for fitting a source model. Poor focal sphere coverage can further contribute to uncertainty. Here, we focus on the statistical trend of these values for a large data set, rather than the values for any given event. The trends of the curves shown in Fig. 4 indicate systematic variations with uncertainties much smaller than the uncertainty in the attributes of any single event. The standard deviation of the residuals about the curve is about 0.19 magnitude units (Table 1). This value should be kept in mind when converting the size of events from $M_{\mathrm{L}}$ into $P_{0}, M_{0}$ or $M_{\mathrm{w}}$, especially when done for individual events. However, converting an entire earthquake catalogue with $M_{\mathrm{L}}$ values into $P_{0}, M_{0}$ or $M_{\mathrm{w}}$, and performing a statistical analysis on the results, should be more robust since much of this variability will average out.

Local magnitude has been a valuable metric for quantifying the size of earthquakes for more than half a century because of its ease of calculation and general stability. However, the local magnitudes saturate for large events and can underestimate the size of small events because of attenuation and recording issues. The moment magnitude scale was introduced several decades ago, but it is still used primarily only for moderate to large earthquakes. The scaling relation of Hanks \& Kanamori (1979) does not characterize the relation between moments and local magnitudes of small events. All these issues have the potential of producing errors and biases in analyses of $b$-values or other parameters of earthquake catalogues. As another example, we note that in calculating spectral ratios for extracting source properties of earthquakes, a commonly employed rule of thumb is to use an empirical Green's function from events (e.g. Hough \& Dreger 1995) that are 1-2 units smaller than the main earthquake to be studied. This rule of thumb was probably meant for moment magnitude, not local magnitude, and the results obtained in this study indicate using $M_{\mathrm{L}}$ for this determination may lead to empirical Green's functions with corner frequencies that are considerably lower than expected.

The source of the discrepancy between local magnitudes and moment magnitudes has been discussed by several studies in the past. Bakun (1984) and Hanks \& Boore (1984) attributed the deviation to the Wood-Anderson filter that is applied to seismograms in determining local magnitude. The Wood-Anderson filter has a 
corner frequency of roughly $1 \mathrm{~Hz}$, and artificially lowers the amplitude of larger events more than smaller events. Edwards et al. (2010) pointed out that a similar effect can be produced by seismic attenuation which strongly affects the higher frequencies of smaller events more than the lower frequencies of larger events. The stronger effect of attenuation on wavefields radiated by smaller events is illustrated in figs 11 and 12 of Kwiatek \& Ben-Zion (2016) with synthetic calculations. The divergence between the $M_{\mathrm{L}}$ and $M_{\mathrm{w}}$ scales of small events may also be related to the fact that they propagate in a very heterogeneous stress field (which prevents them from becoming larger), in contrast to moderate and large events that propagate in a more uniform stress field (e.g. Ben-Zion 1996). The limiting scaling relation between the potency and rupture area in a highly heterogeneous stress field is $P_{0} \sim A$ (Fisher et al. 1997), while crack-like events propagating in a uniform stress field satisfy the classical scaling relation $P_{0} \sim A^{3 / 2}$ (e.g. Ben-Zion 2008). Therefore, the $3 / 2$ scaling parameter in the moment-magnitude scaling relation of Hanks \& Kanamori (1979) is expected to hold for moderate and large events, but a smaller scaling parameter is expected theoretically for small events. This can contribute to the discrepancy between $M_{\mathrm{L}}$ and $M_{\mathrm{W}}$ of small events (Ben-Zion \& Zhu 2002). In any case, the differences between the local and moment magnitudes should be kept in mind when analysing earthquake data sensitive to the scale.

\section{ACKNOWLEDGEMENTS}

The study was supported by the Earthquake Hazards Programme of the United States Geological Survey (grant G15AP00084). The paper benefitted from reviews by Nicholas Deichmann, two anonymous reviewers, and editor Jörg Renner.

\section{REFERENCES}

Abercrombie, R.E., 1995. Earthquake source scaling relationships from -1 to $5 M_{\mathrm{L}}$ using seismograms recorded at $2.5-\mathrm{km}$ depth, J. geophys. Res., 100, 24 015-24 036.

Abercrombie, R.E., 1996. The magnitude-frequency distribution of earthquakes recorded with deep seismometers at Cajon Pass, southern California, Tectonophysics, 261, 1-7.

Abercrombie, R.E., 1997. Near-surface attenuation and site effects from comparison of surface and deep borehole recordings, Bull. seism. Soc. Am., 87, 731-744.

Aki, K., 1965. Maximum likelihood estimate of $\mathrm{b}$ in the formula $\log \mathrm{N}=\mathrm{a}-$ $\mathrm{bM}$ and its confidence limits, Bull. Earthq. Res. Inst., Tokyo Univ., 43, 237-239.

Aki, K., 1966. Generation and propagation of $\mathrm{G}$ waves from the Niigata Earthquake of June 16, 1964: Part 2. Estimation of earthquake moment, released energy, and stress-strain drop from the G wave spectrum, Bull. Earthq. Res. Inst., 44, 73-88.

Aki, K. \& Richards, P.G., 2002. Quantitative Seismology, 2nd edn, University Science Books.

Allam, A. \& Ben-Zion, Y., 2012. Seismic velocity structures in the Southern California plate-boundary environment from double-difference tomography, Geophys. J. Int., 190, 1181-1196.

Allen, R., 1978. Automatic earthquake recognition and timing from single traces, Bull. seism. Soc. Am., 68, 1521-1532.

Allen, R., 1982. Automatic phase pickers: their present use and future prospects, Bull. seism. Soc. Am., 72, S225-S242.

Bakun, W.H., 1984. Seismic moments, local magnitudes, and coda-duration magnitudes for earthquakes in central California, Bull. seism. Soc. Am., 74, 439-458.

Ben-Menahem, A. \& Singh, S.J., 2012. Seismic Waves and Sources, Springer Science and Business Media.
Ben-Zion, Y., 1996. Stress, slip and earthquakes in models of complex single-fault systems incorporating brittle and creep deformations, J. geophys. Res., 101, 5677-5706.

Ben-Zion, Y., 2003. Appendix 2: key formulas in earthquake seismology, in International Handbook of Earthquake and Engineering Seismology, Part B, pp. 1857-1875, eds Lee, W.H.K., Kanamori, H., Jennings, P.C. \& Kisslinger, C., Academic Press.

Ben-Zion, Y., 2008. Collective behavior of earthquakes and faults: continuum-discrete transitions, progressive evolutionary changes and different dynamic regimes, Rev. Geophys., 46, RG4006, doi:10.1029/2008RG000260.

Ben-Zion, Y. \& Zhu, L., 2002. Potency-magnitude scaling relations for southern California earthquakes with $1.0<M_{\mathrm{L}}<7.0$, Geophys. J. Int., 148, F1-F5.

Boore, D.M. \& Boatwright, J., 1984. Average body-wave radiation coefficients, Bull. seism. Soc. Am., 74, 1615-1621.

Brune, J.N., 1970. Tectonic stress and the spectra of seismic shear waves from earthquakes, J. geophys. Res., 75(26), 4997-5009.

Deichmann, N., 2006. Local magnitude, a moment revisited, Bull. seism. Soc. Am., 96, 1267-1277.

Edwards, B., Allmann, B., Fah, D. \& Clinton, J., 2010. Automatic computation of moment magnitudes for small earthquakes and the scaling of local to moment magnitude, Geophys. J. Int., 183, 407-420.

Fisher, D.S., Dahmen, K., Ramanathan, S. \& Ben-Zion, Y., 1997. Statistics of earthquakes in simple models of heterogeneous faults, Phys. Rev. Lett., 78, 4885-4888.

Gerstenberger, M., Wiemer, S. \& Giardini, D., 2001. A systematic test of the hypothesis that the b value varies with depth in California, Geophys. Res. Lett., 28, 57-60.

Gutenberg, B. \& Richter, C.F., 1944. Frequency of earthquakes in California, Bull. seism. Soc. Am., 34, 185-188.

Hanks, T.C. \& Boore, D.M., 1984. Moment-magnitude relations in theory and practice, J. geophys. Res., 89, 6229-6235.

Hanks, T.C. \& Kanamori, H., 1979. A moment magnitude scale, J. geophys. Res., 84(B5), 2348-2350.

Hough, S. \& Dreger, D., 1995. Source parameters of the 23 April 1992 M 6.1 Joshua Tree, California, earthquake and its aftershocks: empirical Green's function analysis of GEOS and TERRAscope data, Bull. seism. Soc. Am., 85, 1576-1590.

Ide, S., Beroza, G.C., Prejean, S.G. \& Ellsworth, W.L., 2003. Apparent break in earthquake scaling due to path and site effects on deep borehole recordings, J. geophys. Res., 108(B5), doi:10.1029/2001JB001617.

Kilb, D., Biasi, G., Anderson, J.G., Brune, J., Peng, Z. \& Vernon, F.L., 2012. A comparison of spectral parameter kappa from small and moderate earthquakes using southern California ANZA seismic network data, Bull. seism. Soc. Am., 102, 284-300.

Kwiatek, G. \& Ben-Zion, Y., 2016. Theoretical limits on detection and analysis of small earthquakes, J. geophys. Res., 121, doi:10.1002/2016JB012908.

Liu, X., Ben-Zion, Y. \& Zigone, D., 2015. Extracting seismic attenuation coefficients from cross-correlations of ambient noise at linear triplets of stations, Geophys. J. Int., 203, 1149-1163.

Park, J., Lindberg, C.R. \& Vernon, F.L., 1987. Multitaper spectral analysis of high-frequency seismograms, J. geophys. Res., 92(B12), 12 675-12 684.

Prieto, G.A., Shearer, P.M. \& Vernon, F.L., 2004. Earthquake source scaling and self-similarity estimation from stacking $\mathrm{P}$ and S spectra, J. geophys. Res., 109, B08310, doi:10.1029/2004JB003084.

Richter, C.F., 1935. An instrumental earthquake magnitude scale, Bull. seism. Soc. Am., 25, 1-32.

Ross, Z.E. \& Ben-Zion, Y., 2014. Automatic picking of direct P, S seismic phases and fault zone head waves, Geophys. J. Int., 199(1), 368-381.

Ross, Z.E. \& Ben-Zion, Y., 2016. Towards reliable automated estimates of earthquake source properties from body wave spectra, J. geophys. Res., 121, doi:10.1002/2016JB013003.

Ross, Z.E., White, M.C., Vernon, F.L. \& Ben-Zion, Y., 2016. An improved algorithm for real-time $S$-wave picking with application to the (augmented) ANZA network in southern California, Bull. seism. Soc. Am., 106, doi:10.1785/0120150230. 
SCEDC, 2013. Southern California Earthquake Center, Caltech. Dataset. doi:10.7909/C3WD3xH1

Shearer, P.M., Prieto, G.A. \& Hauksson, E., 2006. Comprehensive analysis of earthquake source spectra in southern California, J. geophys. Res., 111, B06303, doi:10.1029/2005JB003979.

Thomson, D.J., 1982. Spectrum estimation and harmonic analysis, Proc. IEEE, 70, 1055-1096.

Utsu, T., 1999. Representation and analysis of the earthquake size distribution: a historical review and some new approaches, in Seismicity Patterns, their Statistical Significance and Physical Meaning, pp. 509-535, eds Wyss, M., Shimazaki, K. \& Ito, A., Springer.

Waldhauser, F. \& Ellsworth, W.L., 2000. A double-difference earthquake location algorithm: method and application to the northern Hayward fault, California, Bull. seism. Soc. Am., 90, 1353-1368.

\section{SUPPORTING INFORMATION}

Additional Supporting Information may be found in the online version of this paper:

Table S1. Velocity and $Q$ model used for potency calculations. (http://gji.oxfordjournals.org/lookup/suppl/doi:10.1093/gji/ ggw327/-/DC1)

Please note: Oxford University Press is not responsible for the content or functionality of any supporting materials supplied by the authors. Any queries (other than missing material) should be directed to the corresponding author for the paper. 